Supplement of The Cryosphere, 12, 2901-2922, 2018

https://doi.org/10.5194/tc-12-2901-2018-supplement

(C) Author(s) 2018. This work is distributed under

the Creative Commons Attribution 4.0 License.

(c) (1)

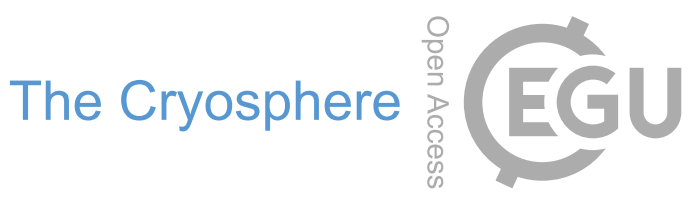

Supplement of

\title{
Melting over the northeast Antarctic Peninsula (1999-2009): evaluation of a high-resolution regional climate model
}

Rajashree Tri Datta et al.

Correspondence to: Rajashree Tri Datta (tri.datta@gmail.com)

The copyright of individual parts of the supplement might differ from the CC BY 4.0 License. 


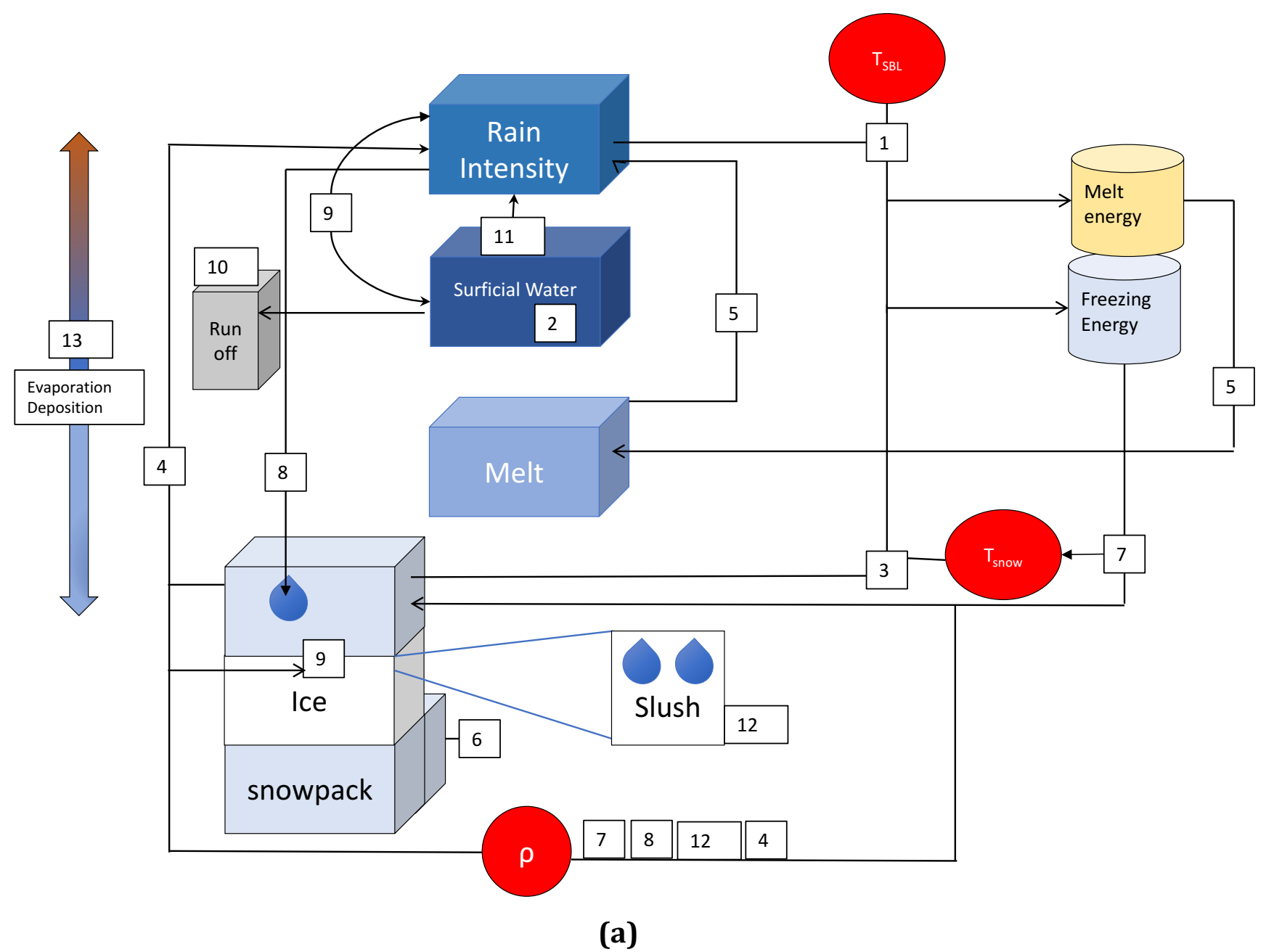




\section{Step}

1) Energy from Rain

2) Surficial Water Exists? (a function of $T_{\text {exc }}$ )

3) Energy from Snow

Energy $<-\rho * C s * T_{\text {exc }} * d s$

4) Water from snowpack

$\mathrm{RI}<-\rho^{*} \mathrm{ds} *$ (soil humidity) $\rho$ reduced by soil humidity

5) MELT (when Energy is positive)

melted snow <- Energy / (Lf* $\rho$ )

$$
\text { ds } \rightarrow>\text { melted snow } \rightarrow \text { RI }
$$

6) Alter the snow history based on whether melting is occurring

faceted crystals? Liquid water with no faceted crystals?

Liquid water with faceted crystals before?

7) FREEZE (when Energy is negative)

Energy / (Lf* $\rho$ ) <- frozen water <- R

Tsnow $\rightarrow$ Energy / $(\rho *$ ds $*$ Cs $)$

$\rho$ increased by the addition of frozen water

8) Water saturation in snow

an irreducible portion of the snowpack must contain water. $\mathrm{RI}->$ irreducible water in snowpack (constant * pore volume * ds * density(water) irreducible water in snowpack $\rightarrow \rho$

$* * *$ denotes steps where tuning is possible or separate physics are calculated depending on the region

\begin{tabular}{|ll}
\hline & Variables \\
& Rain Intensity \\
$\mathrm{RI}_{\text {exc }}$ & Temp above/below 0 \\
$\mathrm{t}$ & Time elapsed \\
$\mathrm{\rho}$ & density snow \\
$\mathrm{ds}$ & snow depth \\
$\mathrm{LHF}$ & latent heat flux $\mathrm{W} \mathrm{m}^{-2}$ \\
\hline
\end{tabular}

Energy +or -

Energy +or

Rain Intensity + density -

Energy -

snow depth -

Temperature -

Rain Intensity -

Energy +

Density +

Intensity
Density +
Energy $<-\mathrm{RI} * \mathrm{CW}^{*} \mathrm{~T}_{\text {exc }} * \mathrm{t}$

\section{Step}

9) Pore hole close off / superimposed ice

Effect on Variables

Surficial Water, Rain Intensity + / -

ice and a constant value for pore hole close off density.

Pore close off $->\mathrm{RI}$ converted to surficial wate

No pore close off $->\mathrm{RI}$ remains in $\mathrm{RI}$

\section{0) Surficial water runoff}

Final Energy reduced by Energy from rain (Step 1)

Surficial water -

A decay function determines the portion of surficial water converted to runoff Reference: Zuo and Oerlemans, 1996

\section{1) Conversion back to rain}

Surficial water-

added back into RI (rain intensity)

\section{2) Slush}

Where surficial water exists (step 2), the highest snow/ice layer will fill the pore volume with water from surficial water, adding to density slush $+<-$ surficial water slush $\rightarrow p+$

Surficial water density +

\section{3) Add/Subtract Deposition/Sublimation}

Snowpack either + /

DepOrSubl= $t *$ LHF $/\left(L x^{*} \rho\right)<->d s$ added to the snowpack

$\operatorname{EnVp}=\left(C s * T_{\text {exc }}\right.$-Lf $*(1$-soil humidity $\left.)\right) /(1+($ DepOrSubl / ds $))$

Soil/Ice Humidity + /Temp $+/-$

Hum $=1+(E n p$ ased to alter humidity of soil/snow

Hum $=1+\left(E n V p-\right.$ Texc $\left.^{*} C s\right) /$ Lf

as well as the tem
$T_{\text {exc }}=\left(E n V p+L f^{*}(1-H u m)\right) / C s$

$\begin{array}{llll} & & \text { Constants: } & \\ \mathrm{kg} \mathrm{m}^{-2} \mathrm{~s}^{-1} & \mathrm{Cw} & \text { Heat Capacity of Water } & \mathrm{Jkg}^{-1} \mathrm{~K}^{-1} \\ \mathrm{C} & \mathrm{Cs} & \text { Heat Capacity of snow } & \mathrm{Jkg}^{-1} \mathrm{~K}^{-1} \\ \mathrm{~s} & \mathrm{Lf} & \text { Latent heat of fusion } & \mathrm{Jkg}^{-1} \\ \mathrm{~kg} \mathrm{~m} & \mathrm{Lx} & \text { Latent Heat of Vap/Subl } & \mathrm{Jkg}^{-1}\end{array}$

\section{(b)}

Figure S1: Diagram (a) and description (b) of the physical processes within MAR's SISVAT (Soil Ice Vegetation Atmosphere Transfer Scheme) calculating meltwater production and meltwater percolation into the snowpack from the energy balance and the presence of water, using the density of the snowpack ( $\rho$ ), temperature of the surface boundary layer (TSBL) and temperature of the snow (TSNOW). 


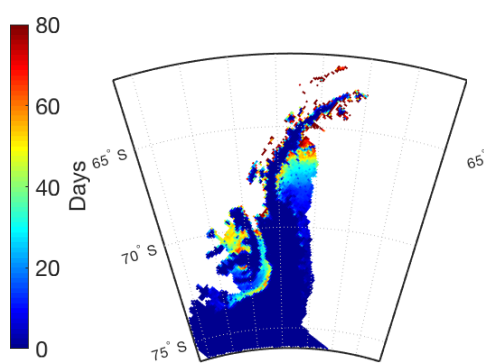

(a) $0.1 \mathrm{~mm}$ w.e.

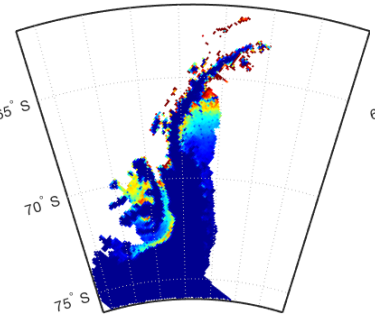

(b) $0.4 \mathrm{~mm}$ w.e.

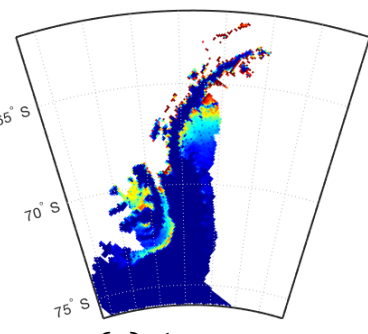

(c) $1 \mathrm{~mm}$ w.e.

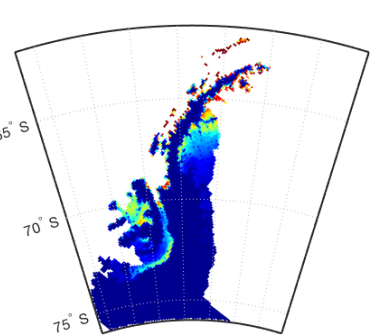

(d) $4 \mathrm{~mm}$ w.e.

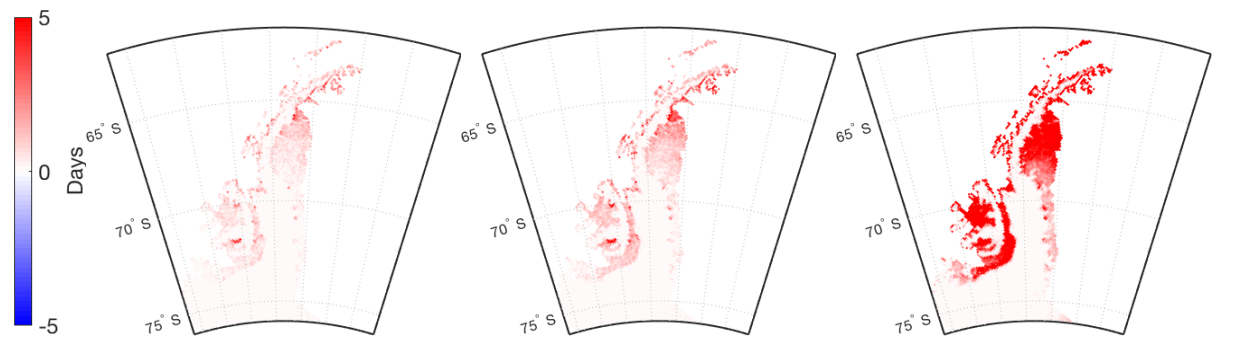

(e) $0.1-0.4$

(f) $1-0.4$

(g) 4-1

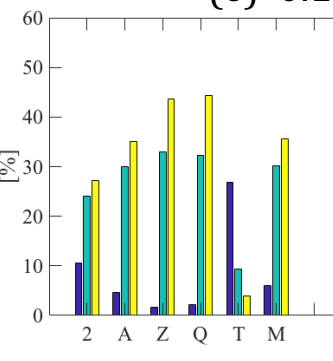

Treshhol

d

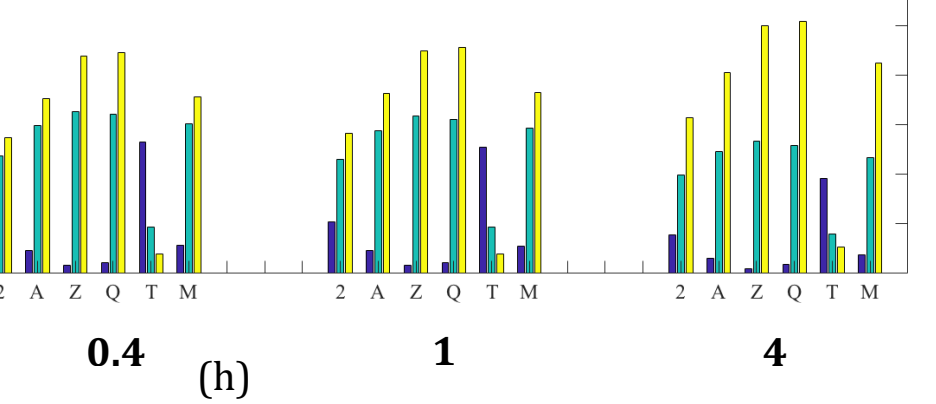

(h)

4

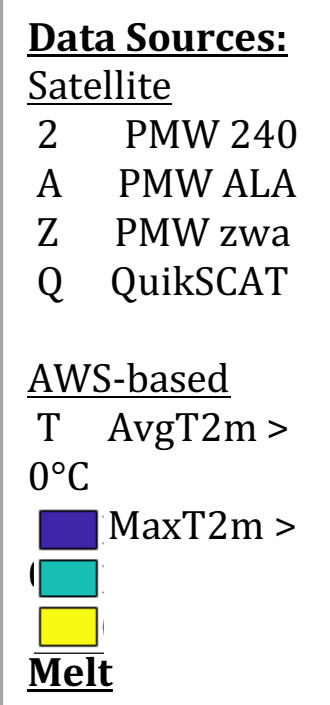

Figure S2: Average MAR melt duration from 2000-2009 using different thresholds for total daily meltwater production to determine melt occurrence (1) 0.1 mm w.e. (b) $0.4 \mathrm{~mm}$ w.e. (c) $1 \mathrm{~mm}$ w.e. (d) $4 \mathrm{~mm}$ w.e. Major differences in average yearly melt duration between melt thresholds (e) $0.1 \mathrm{~mm}$ w.e. $-0.4 \mathrm{~mm}$ w.e. (f) $1 \mathrm{~mm}$ w.e. $-0.4 \mathrm{~mm}$ w.e. (g) $4 \mathrm{~mm}$ w.e. $-1 \mathrm{~mm}$ w.e. 


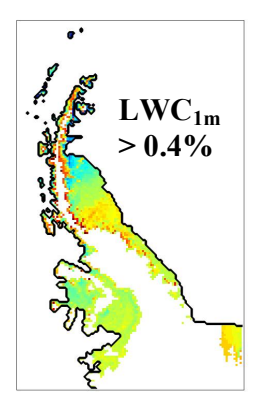

(a)

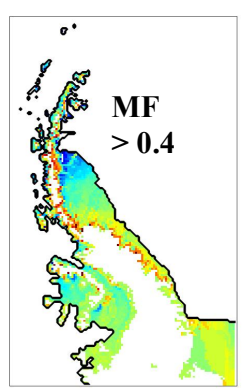

(b)

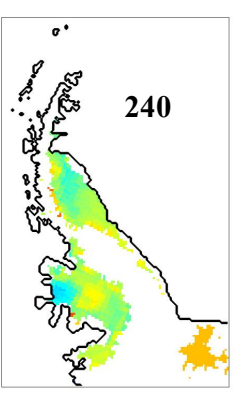

(c)

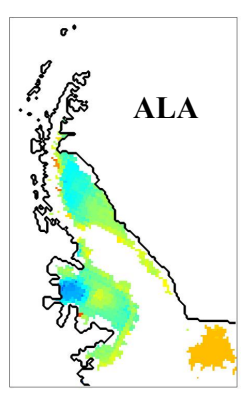

(d)

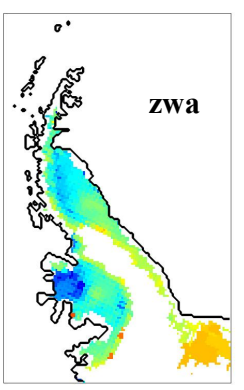

(e)

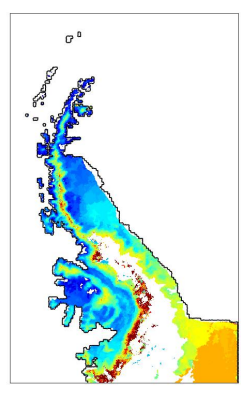

(f)

Figure S3: Average Melt Onset date from multiple sources (a) MAR, Liquid Water Content $>0.4 \%$ for three consecutive days. (b) MAR Total Melt Flux $>0.4$ mmwe for 1 day or more. Satellite-based: (c) PMW 240 algorithm (d) PMW ALA (e) PMW Zwa (f) QuikSCAT. Day shown is the first day of a sustained three-day melt period for satellite estimates as well as $\mathrm{LWC}_{1 \mathrm{~m}}$, Date number is defined beginning in $\mathrm{Jan} 1^{\text {st }}$. of year1, such that 365 represents Dec $31^{\text {st }}$ of year1. All averages are taken from the 2000-2009 period to retain consistency with the availability of QuikSCAT data.

12

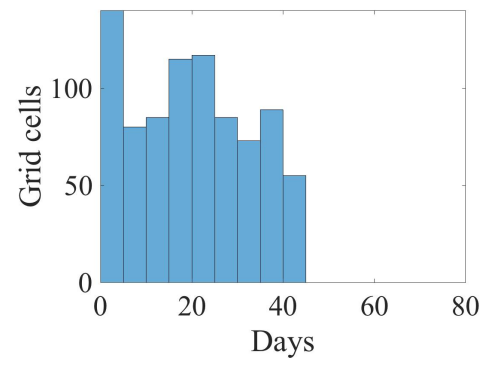

(a)

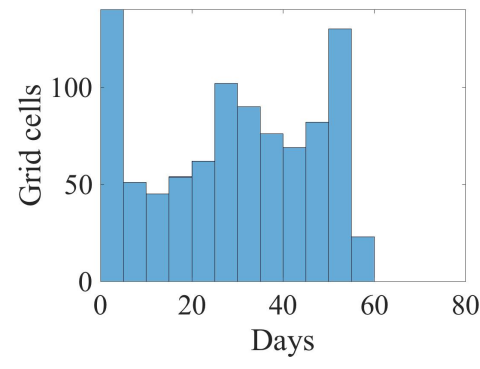

(b)

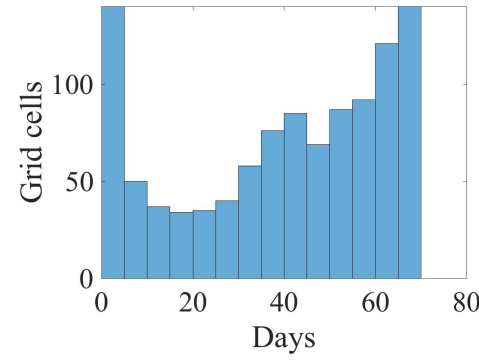

(c)

Figure S4: Number of 10km MAR grid cells from the NE basin (y axis) showing the avg number of total melt days (2001- 


\begin{tabular}{|c|c|c|c|}
\hline Region & $\begin{array}{c}\text { Avg. Annual Melt } \\
\text { Days } \\
(2001-2014) \\
\text { [Days] }\end{array}$ & $\begin{array}{c}\text { Elevation } \\
{[\mathrm{m}]}\end{array}$ & $\begin{array}{l}\text { Avg coincident } \\
\text { MAR Meltwater } \\
\text { Production } \\
\text { NDJF } \\
\text { (2001 to 2014) } \\
{\left[\mathrm{mmWE} / 100 \mathrm{~km}^{2}\right]}\end{array}$ \\
\hline $240 \mathrm{~L}$ & $1 \leq \mathrm{D}<10$ & $833.70 \pm 539.62$ & 7.81 \\
\hline $240 \mathrm{M}$ & $10 \leq \mathrm{D}<30$ & $72.37 \pm 90.98$ & 55.32 \\
\hline $240 \mathrm{H}$ & $30 \leq=\mathrm{D}$ & $42.94 \pm 17.78$ & 95.09 \\
\hline ALA L & $1 \leq \mathrm{D}<15$ & $1016.13 \pm 525.80$ & 7.28 \\
\hline ALA M & $15 \leq \mathrm{D}<40$ & $125.97 \pm 200.67$ & 62.94 \\
\hline ALA H & $40 \leq=\mathrm{D}$ & $56.92 \pm 56.69$ & 128.72 \\
\hline zwa L & $1 \leq \mathrm{D}<20$ & $1165.99 \pm 513.24$ & 7.82 \\
\hline zwa M & $20 \leq \mathrm{D}<45$ & $374.80 \pm 471.47$ & 47.55 \\
\hline zwa H & $45 \leq \mathrm{D}$ & $101.73 \pm 173.27$ & 126.19 \\
\hline CL Region & & $42.67 \pm 17.68$ & \\
\hline PMW All & $36.63 \pm 4.01$ & $39.15 \pm 17.87$ & 96.15 \\
\hline $\mathrm{MF}_{0.4}$ & $21.29 \pm 9.10$ & $42.15 \pm 16.05$ & 143.08 \\
\hline NL Region & & $594.12 \pm 601.20$ & \\
\hline PMW All & $7.74 \pm 8.90$ & $86.72 \pm 137.87$ & 41.24 \\
\hline $\mathrm{MF}_{0.4}$ & $26.68 \pm 24.94$ & $126.88 \pm 159.87$ & 231.97 \\
\hline
\end{tabular}

2 Table S1: Average statistics for regions of melt occurrence, restricted to the NE basin. The first 9 rows indicate regions

3 where melt occurrence is determined by a PMW algorithm (i.e. 240) restricted by the number of days where melt occurrence (i.e. $240 \mathrm{~L}$, where the number of avg annual melt days is between 1 and 10). CL and NL regions are described in text. Row indicating "PMW All"or "MF ${ }_{0.4}$ " in left column implies that corresponding statistics in columns 2-4 are calculated for where melt occurrence meets these conditions 
1

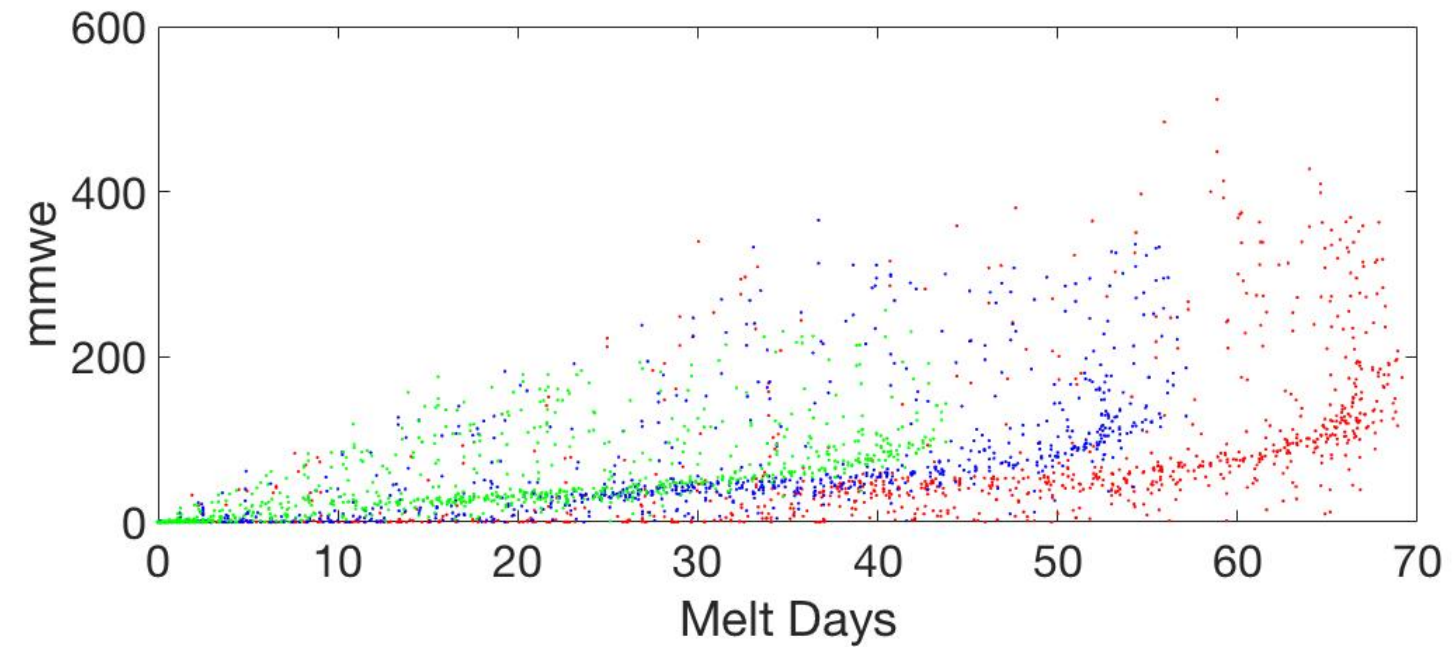

3

4 Figure S5: Avg Melt Days (2001-2014) from three passive microwave algorithms (described in text). Green shows PMW 5 240. Blue shows PMW ALA. Red shows PMW zwa. 


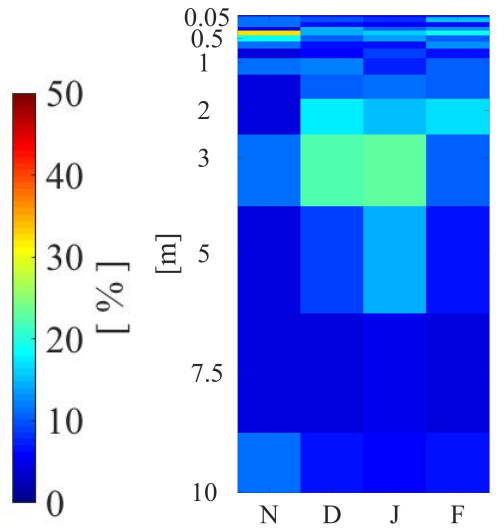

(a) $240-\mathrm{L}$

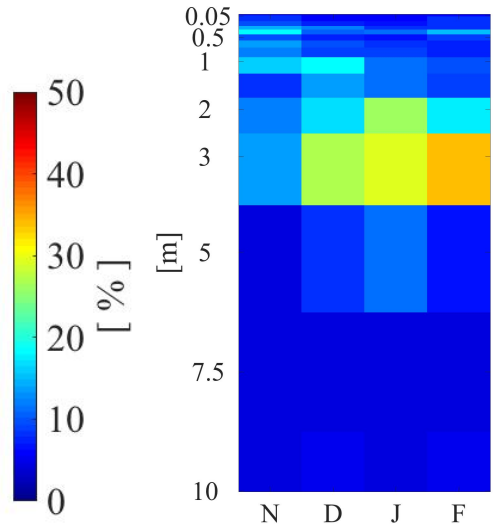

(d) $240-\mathrm{M}$

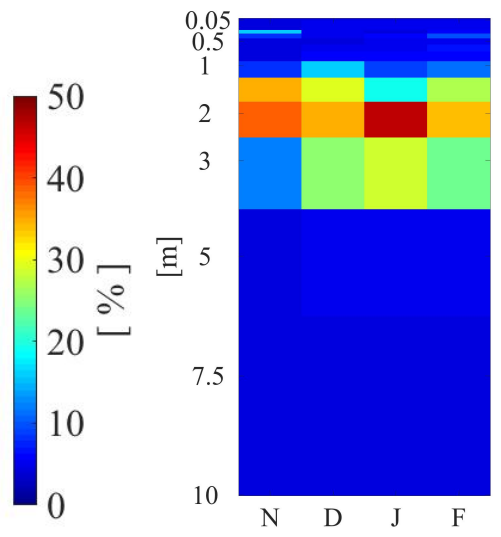

(g) $240-\mathrm{H}$

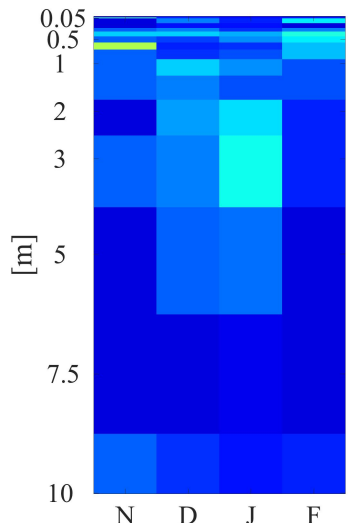

(b) ALA-L

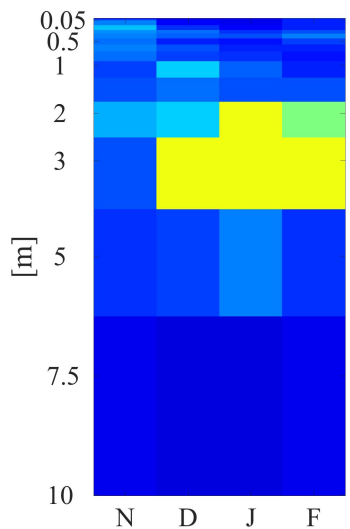

(e) ALA-M

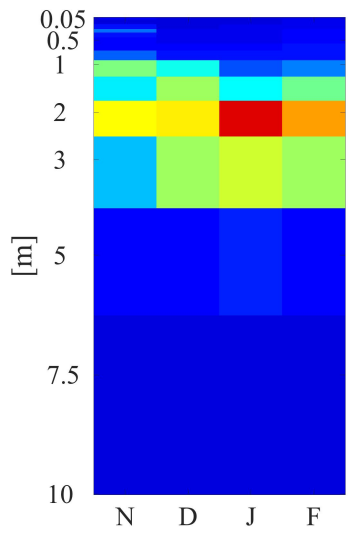

(h) ALA-H

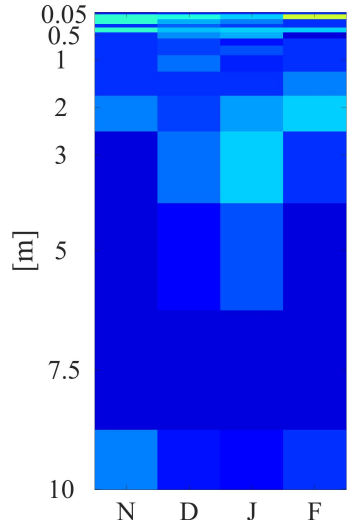

(c) zwa-L

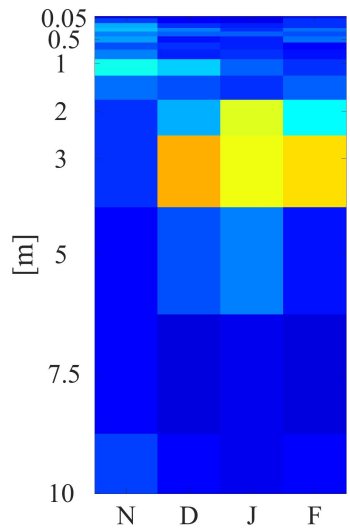

(f) zwa-M

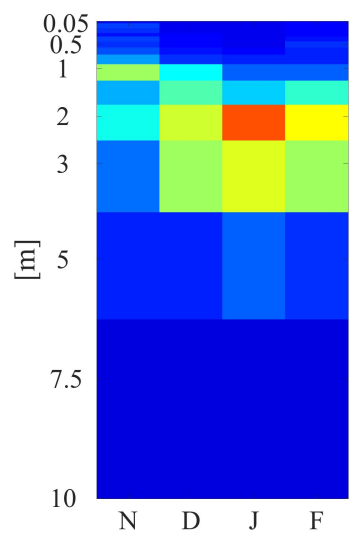

(i) zwa-H

Figure S6: Maximum depth of MAR-modeled meltwater percolation (MAR) into the snowpack over the melt season. Colors indicate the percentage of grid cells where meltwater reaches the corresponding maximum depth ( $y$ axis) for the month ( $x$ axis), such that each column per month totals to $100 \%$. Maximum percolation depth is determined by the maximum depth over the month where liquid water content in MAR is greater than $0.02 \mathrm{~kg} / \mathrm{kg}$. Grid cells for each column are restricted to the corresponding month during the 2001-2014 period which fulfill the conditions (a) 240-L (b) ALA-L (c) zwa-L (d)240-M (e) ALA-M (f) zwa-M (g) 240-H (h) ALA-H (i) zwa-H, as defined in table 1. 
1

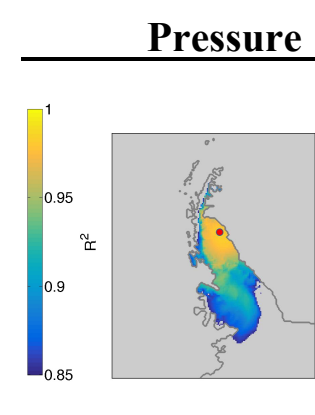

(a)

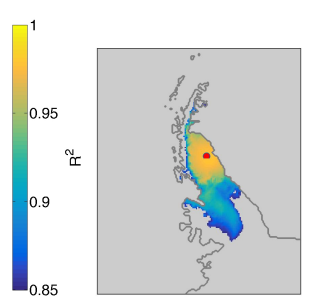

(d)

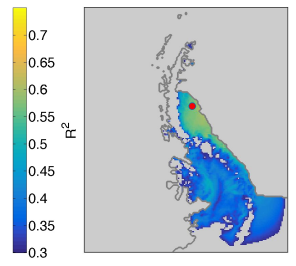

(b)

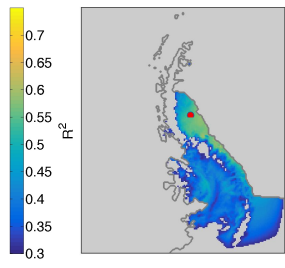

(e)

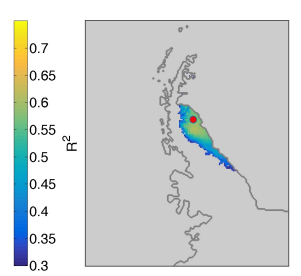

(c)

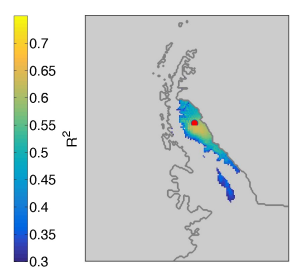

(f)

7

8 Figure S7: Left column is for surface pressure. Middle column for daily-averaged $2 \mathrm{~m}$ air temperature, right column for

$92 \mathrm{~m}$ wind speed. Stations are as follows: (a)(b)(c) AWS 14/Larsen Ice Shelf, which are co-located in MAR (d)(e)(f) AWS 15 


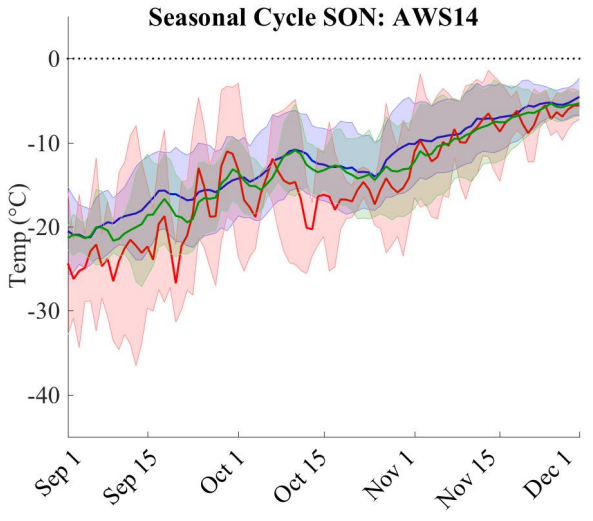

(a)

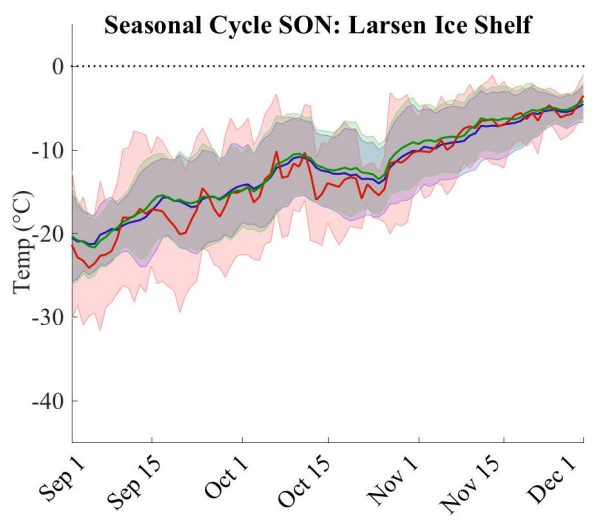

(c)

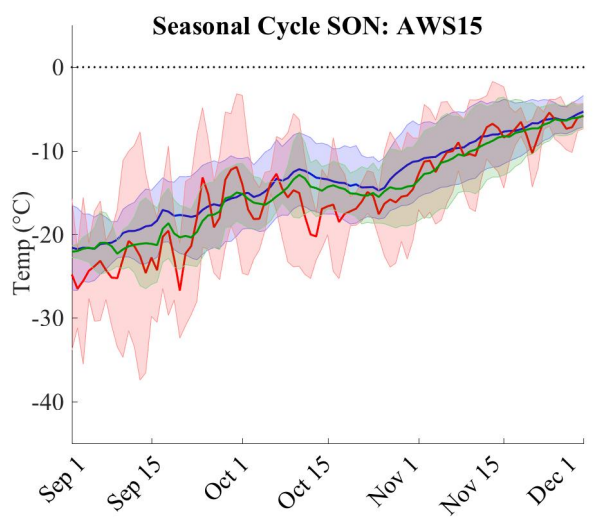

(e)

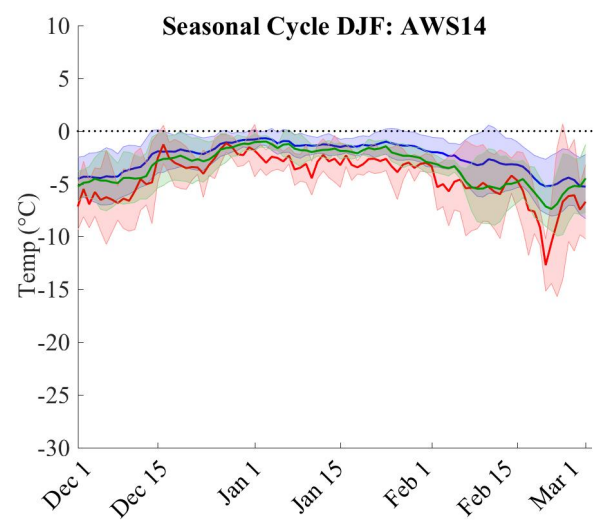

(b)

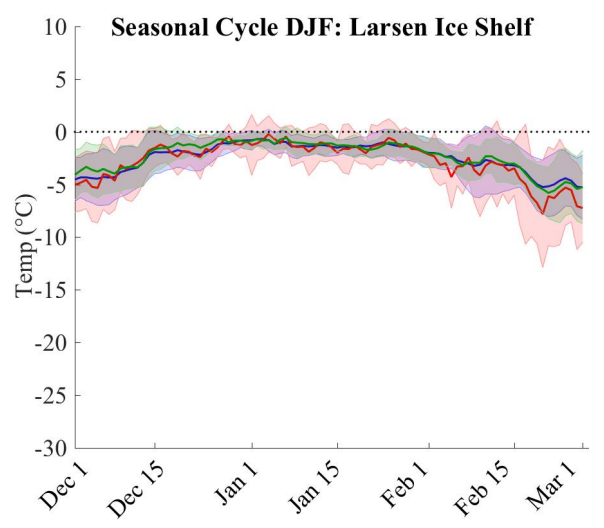

(d)

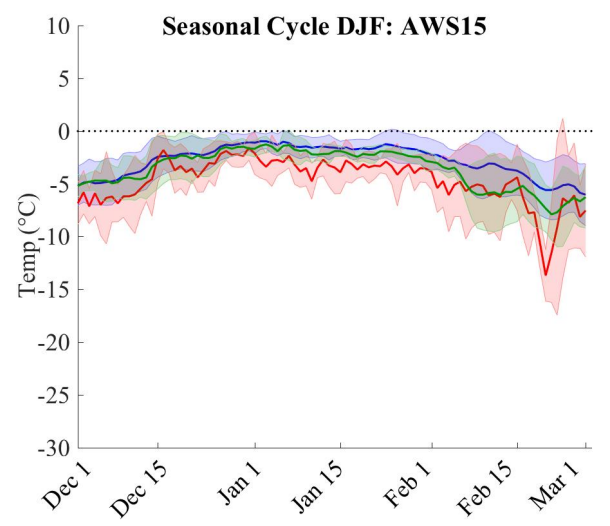

(f)

Figure S8: Seasonal Avg Ts climatology for spring (SON) and summer(DJF) with envelope indicating one standard deviation, Red: computed for available data from AWS station, with quality control as described in section 2. Green: MAR daily-averaged T2m data restricted to AWS-data availability. Blue: MAR daily-averaged T2m data for the full period (1999-2014). Data is shown for (a)(b) AWS 14 (c)(d) Larsen Ice Shelf (e)(f) AWS 15

\section{3}




\begin{tabular}{|l|l|l|l|l|l|l|l|l|l|l|l|l|l|}
\hline & $\mathbf{0 1 - 0 2}$ & $\mathbf{0 2 - 0 3}$ & $\mathbf{0 3 - 0 4}$ & $\mathbf{0 4 - 0 5}$ & $\mathbf{0 5 - 0 6}$ & $\mathbf{0 6 - 0 7}$ & $\mathbf{0 7 - 0 8}$ & $\mathbf{0 8 - 0 9}$ & $\mathbf{0 9 - 1 0}$ & $\mathbf{1 0 - 1 1}$ & $\mathbf{1 1 - 1 2}$ & $\mathbf{1 2 - 1 3}$ & $\mathbf{1 3 - 1 4}$ \\
\hline AWS14 & & & & & & & & & 0.98 & 0.98 & 0.98 & 0.99 & 0.97 \\
\hline AWS15 & & & & & & & & & 0.98 & 0.98 & & 0.60 & 0.97 \\
\hline Larsen IS & 0.99 & 0.98 & 0.98 & & & & 0.98 & 0.98 & 0.98 & 0.98 & 0.98 & & \\
\hline
\end{tabular}

Table S2: $\mathrm{R}^{2}$ values between MAR and AWS data for Surface Pressure in Summer (DJF) for years shown

\begin{tabular}{|l|l|l|l|l|l|l|l|l|l|l|l|l|l|}
\hline & $\mathbf{0 1 - 0 2}$ & $\mathbf{0 2 - 0 3}$ & $\mathbf{0 3 - 0 4}$ & $\mathbf{0 4 - 0 5}$ & $\mathbf{0 5 - 0 6}$ & $\mathbf{0 6 - 0 7}$ & $\mathbf{0 7 - 0 8}$ & $\mathbf{0 8 - 0 9}$ & $\mathbf{0 9 - 1 0}$ & $\mathbf{1 0 - 1 1}$ & $\mathbf{1 1 - 1 2}$ & $\mathbf{1 2 - 1 3}$ & $\mathbf{1 3 - 1 4}$ \\
\hline AWS14 & & & & & & & & & 2.36 & 1.71 & 2.36 & 2.4 & 2.48 \\
\hline AWS15 & & & & & & & & & 2.46 & 2.05 & & 6.04 & 1.97 \\
\hline Larsen IS & 1.14 & 1.71 & 2.01 & & & & 2.07 & 2.25 & 2.25 & 1.77 & 2.19 & & \\
\hline
\end{tabular}

Table S3: Root Mean Squared Error between MAR and AWS data for Pressure [hPa] in Summer (DJF) for years shown

\begin{tabular}{|l|l|l|l|l|l|l|l|l|l|l|l|l|l|}
\hline & $\mathbf{0 1 - 0 2}$ & $\mathbf{0 2 - 0 3}$ & $\mathbf{0 3 - 0 4}$ & $\mathbf{0 4 - 0 5}$ & $\mathbf{0 5 - 0 6}$ & $\mathbf{0 6 - 0 7}$ & $\mathbf{0 7 - 0 8}$ & $\mathbf{0 8 - 0 9}$ & $\mathbf{0 9 - 1 0}$ & $\mathbf{1 0 - 1 1}$ & $\mathbf{1 1 - 1 2}$ & $\mathbf{1 2 - 1 3}$ & $\mathbf{1 3 - 1 4}$ \\
\hline AWS14 & & & & & & & & & -2.10 & -1.32 & -2.04 & -2.26 & -2.17 \\
\hline AWS15 & & & & & & & & & -2.21 & -1.72 & & -0.84 & -1.59 \\
\hline Larsen IS & -0.65 & -1.21 & -1.66 & & & & -1.59 & -1.82 & -1.95 & -1.36 & -1.83 & & \\
\hline
\end{tabular}

Table S4: Mean Error (MAR-AWS) for Pressure [hPa] in Summer (DJF) for years shown

\begin{tabular}{|l|l|l|l|l|l|l|l|l|l|l|l|l|l|}
\hline & $\mathbf{0 1 - 0 2}$ & $\mathbf{0 2 - 0 3}$ & $\mathbf{0 3 - 0 4}$ & $\mathbf{0 4 - 0 5}$ & $\mathbf{0 5 - 0 6}$ & $\mathbf{0 6 - 0 7}$ & $\mathbf{0 7 - 0 8}$ & $\mathbf{0 8 - 0 9}$ & $\mathbf{0 9 - 1 0}$ & $\mathbf{1 0 - 1 1}$ & $\mathbf{1 1 - 1 2}$ & $\mathbf{1 2 - 1 3}$ & $\mathbf{1 3 - 1 4}$ \\
\hline AWS14 & & & & & & & & & 0.68 & 0.41 & 0.71 & 0.39 & 0.59 \\
\hline AWS15 & & & & & & & & & 0.66 & 0.50 & 0.63 & 0.35 & 0.58 \\
\hline Larsen IS & 0.36 & 0.27 & 0.56 & & & & 0.57 & 0.67 & 0.70 & 0.44 & 0.71 & & \\
\hline
\end{tabular}

Table S5: $\mathrm{R}^{2}$ values between MAR and AWS data for daily-averaged $2 \mathrm{~m}$ air temperature in Summer (DJF) for years shown

\begin{tabular}{|l|l|l|l|l|l|l|l|l|l|l|l|l|l|}
\hline & $\mathbf{0 1 - 0 2}$ & $\mathbf{0 2 - 0 3}$ & $\mathbf{0 3 - 0 4}$ & $\mathbf{0 4 - 0 5}$ & $\mathbf{0 5 - 0 6}$ & $\mathbf{0 6 - 0 7}$ & $\mathbf{0 7 - 0 8}$ & $\mathbf{0 8 - 0 9}$ & $\mathbf{0 9 - 1 0}$ & $\mathbf{1 0 - 1 1}$ & $\mathbf{1 1 - 1 2}$ & $\mathbf{1 2 - 1 3}$ & $\mathbf{1 3 - 1 4}$ \\
\hline AWS14 & & & & & & & & & 1.91 & 2.19 & 2.56 & 2.60 & 2.47 \\
\hline AWS15 & & & & & & & & & 2.10 & 1.99 & 2.91 & 2.72 & 2.45 \\
\hline Larsen IS & 1.39 & 2.20 & 3.37 & & & & 1.82 & 2.36 & 1.62 & 1.81 & 2.34 & & \\
\hline
\end{tabular}

10 Table S6: Root Mean Squared Error between MAR and AWS data for daily-averaged $2 \mathrm{~m}$ air temperature $\left[{ }^{\circ} \mathrm{C}\right]$ in

\section{Summer (DJF) for years shown}

12

\begin{tabular}{|l|l|l|l|l|l|l|l|l|l|l|l|l|l|}
\hline & $\mathbf{0 1 - 0 2}$ & $\mathbf{0 2 - 0 3}$ & $\mathbf{0 3 - 0 4}$ & $\mathbf{0 4 - 0 5}$ & $\mathbf{0 5 - 0 6}$ & $\mathbf{0 6 - 0 7}$ & $\mathbf{0 7 - 0 8}$ & $\mathbf{0 8 - 0 9}$ & $\mathbf{0 9 - 1 0}$ & $\mathbf{1 0 - 1 1}$ & $\mathbf{1 1 - 1 2}$ & $\mathbf{1 2 - 1 3}$ & $\mathbf{1 3 - 1 4}$ \\
\hline AWS14 & & & & & & & & & 0.98 & 1.00 & 1.61 & 0.32 & 1.30 \\
\hline AWS15 & & & & & & & & & 1.06 & 0.81 & 1.60 & 0.32 & 1.11 \\
\hline Larsen IS & -0.52 & 0.15 & 2.22 & & & & 0.94 & 1.23 & 0.36 & 0.26 & 1.01 & & \\
\hline
\end{tabular}




\begin{tabular}{|c|c|c|c|c|c|c|c|c|c|}
\hline & \multirow[t]{2}{*}{$\begin{array}{l}\text { Pr. } \\
{[\%]}\end{array}$} & \multicolumn{2}{|c|}{$\begin{array}{c}\text { Mean Avg T2m } \\
{\left[{ }^{\circ} \mathrm{C}\right]}\end{array}$} & \multicolumn{2}{|c|}{$\begin{array}{c}\text { Mean MaxT2m } \\
{\left[{ }^{\circ} \mathrm{C}\right]} \\
\end{array}$} & \multicolumn{4}{|c|}{$\begin{array}{c}\text { Temp Bias } \\
{\left[{ }^{\circ} \mathrm{C}\right]}\end{array}$} \\
\hline & & MAR & AWS & MAR & AWS & $\operatorname{AvgT2m}$ & $\begin{array}{l}\operatorname{AvgT2m} \\
>0\end{array}$ & MaxT2m & $\begin{array}{c}\operatorname{MaxT2m} \\
>0\end{array}$ \\
\hline$\overline{A L L}$ & 8 & $\begin{array}{c}-2.31 \\
( \pm 2.27)\end{array}$ & $\begin{array}{c}-3.18 \\
( \pm 3.61)\end{array}$ & $\begin{array}{c}-1.50 \\
( \pm 2.19)\end{array}$ & $\begin{array}{c}0.54 \\
( \pm 3.25)\end{array}$ & $\begin{array}{c}0.88 \\
( \pm 2.87)\end{array}$ & $\begin{array}{c}-1.45 \\
( \pm 1.56)\end{array}$ & $\begin{array}{c}-2.07 \\
( \pm 2.88)\end{array}$ & $\begin{array}{c}-3.36 \\
( \pm 2.23)\end{array}$ \\
\hline MAR & 8 & $\begin{array}{c}-0.70 \\
( \pm 0.80)\end{array}$ & $\begin{array}{c}-1.89 \\
( \pm 2.69)\end{array}$ & $\begin{array}{c}-0.27 \\
( \pm 1.32)\end{array}$ & $\begin{array}{c}1.73 \\
( \pm 2.74)\end{array}$ & $\begin{array}{c}1.19 \\
( \pm 2.53)\end{array}$ & $\begin{array}{c}-0.89 \\
( \pm 1.15)\end{array}$ & $\begin{array}{c}-2.00 \\
2.85( \pm)\end{array}$ & $\begin{array}{c}-3.08 \\
( \pm 2.28)\end{array}$ \\
\hline PMWEx & 6 & $\begin{array}{c}-1.76 \\
( \pm 1.16)\end{array}$ & $\begin{array}{c}-1.73 \\
( \pm 1.63)\end{array}$ & $\begin{array}{c}-0.90 \\
( \pm 1.17)\end{array}$ & $\begin{array}{c}1.45 \\
( \pm 2.19)\end{array}$ & $\begin{array}{c}-0.04 \\
( \pm 1.46)\end{array}$ & $\begin{array}{c}-2.06 \\
( \pm 0.71)\end{array}$ & $\begin{array}{c}-2.35 \\
( \pm 2.12)\end{array}$ & $\begin{array}{c}-2.99 \\
( \pm 1.99)\end{array}$ \\
\hline QSEx & 6 & $\begin{array}{c}-1.91 \\
( \pm 1.18)\end{array}$ & $\begin{array}{c}-2.04 \\
( \pm 2.21)\end{array}$ & $\begin{array}{c}-0.97 \\
( \pm 1.29)\end{array}$ & $\begin{array}{c}1.25 \\
( \pm 2.38)\end{array}$ & $\begin{array}{c}0.12 \\
( \pm 2.03)\end{array}$ & $\begin{array}{c}-2.81 \\
( \pm 1.60)\end{array}$ & $\begin{array}{c}-2.28 \\
( \pm 2.39)\end{array}$ & $\begin{array}{c}-3.15 \\
( \pm 2.15)\end{array}$ \\
\hline
\end{tabular}

\begin{tabular}{|l|c|c|c|c|c|c|c|c|c|}
\hline & $\begin{array}{c}\text { Pr. } \\
{[\%]}\end{array}$ & \multicolumn{2}{|c|}{$\begin{array}{c}\text { Mean Avg T2m } \\
{\left[{ }^{\circ} \mathbf{C}\right]}\end{array}$} & \multicolumn{2}{c|}{$\begin{array}{c}\text { Mean MaxT2m } \\
{\left[{ }^{\circ} \mathbf{C}\right]}\end{array}$} & \multicolumn{4}{c|}{ Temp Bias } \\
{$\left[{ }^{\circ} \mathbf{C}\right]$}
\end{tabular}




\begin{tabular}{|l|c|c|c|c|c|c|c|c|c|}
\hline & $\begin{array}{c}\text { Pr. } \\
{[\%]}\end{array}$ & \multicolumn{2}{|c|}{$\begin{array}{c}\text { Mean Avg T2m } \\
{\left[{ }^{\circ} \mathbf{C}\right]}\end{array}$} & \multicolumn{2}{|c|}{$\begin{array}{c}\text { Mean MaxT2m } \\
{\left[{ }^{\circ} \mathbf{C}\right]}\end{array}$} & \multicolumn{4}{|c|}{ Temp Bias } \\
{$\left[{ }^{\circ} \mathbf{C}\right]$}
\end{tabular}

Table S10: Temp averages and biases, proportions where AWS-observed southesterly winds are preserved in MAR, as a percentage of all wind direction values for the condition. Conditions for ALL, PMWEx and QSEx (in text)

7
8
9

$180-270^{\circ}$ recorded by AWS $90-180^{\circ}$ recorded by MAR

\begin{tabular}{|c|c|c|c|c|c|c|c|c|c|}
\hline & $\begin{array}{l}\text { Pr. } \\
{[\%]}\end{array}$ & \multicolumn{2}{|c|}{$\begin{array}{c}\text { Mean Avg T2m } \\
{\left[{ }^{\circ} \mathrm{C}\right]}\end{array}$} & \multicolumn{2}{|c|}{$\begin{array}{c}\text { Mean MaxT2m } \\
{\left[{ }^{\circ} \mathrm{C}\right]} \\
\end{array}$} & \multicolumn{4}{|c|}{$\begin{array}{c}\text { Temp Bias } \\
{\left[{ }^{\circ} \mathrm{C}\right]}\end{array}$} \\
\hline & & MAR & AWS & MAR & AWS & AvgT2m & $\underset{>0}{\operatorname{AvgT} 2 m}$ & $\operatorname{MaxT2m}$ & $\begin{array}{c}\operatorname{MaxT} 2 \mathrm{~m} \\
>0\end{array}$ \\
\hline ALL & 4 & $\begin{array}{c}-2.13 \\
( \pm 1.77) \\
\end{array}$ & $\begin{array}{c}-3.12 \\
( \pm 3.14) \\
\end{array}$ & $\begin{array}{c}-1.71 \\
( \pm 1.69) \\
\end{array}$ & $\begin{array}{c}-1.01 \\
( \pm 3.39) \\
\end{array}$ & $\begin{array}{c}0.99 \\
( \pm 2.01) \\
\end{array}$ & $\begin{array}{c}-1.27 \\
( \pm 0.59) \\
\end{array}$ & $\begin{array}{c}-0.74 \\
( \pm 2.51)\end{array}$ & $\begin{array}{c}-2.81 \\
( \pm 1.42)\end{array}$ \\
\hline MAR & 4 & $\begin{array}{c}-0.77 \\
( \pm 0.40)\end{array}$ & $\begin{array}{c}-1.27 \\
( \pm 1.20)\end{array}$ & $\begin{array}{c}-0.54 \\
( \pm 0.56)\end{array}$ & $\begin{array}{c}1.35 \\
( \pm 1.98)\end{array}$ & $\begin{array}{c}0.49 \\
( \pm 1.30)\end{array}$ & $\begin{array}{c}-1.21 \\
( \pm 0.57)\end{array}$ & $\begin{array}{c}-1.84 \\
( \pm 2.22)\end{array}$ & $\begin{array}{c}-2.92 \\
( \pm 1.59)\end{array}$ \\
\hline PMWEx & 3 & $\begin{array}{c}-1.69 \\
( \pm 0.62)\end{array}$ & $\begin{array}{c}-1.87 \\
( \pm 1.95)\end{array}$ & $\begin{array}{c}-1.27 \\
( \pm 0.73)\end{array}$ & $\begin{array}{c}-0.32 \\
( \pm 1.62)\end{array}$ & $\begin{array}{c}0.18 \\
( \pm 1.48)\end{array}$ & N/A & $\begin{array}{c}-0.95 \\
( \pm 1.26)\end{array}$ & $\begin{array}{c}-1.98 \\
( \pm 0.56)\end{array}$ \\
\hline QSEx & 4 & $\begin{array}{c}-1.90 \\
( \pm 0.79) \\
\end{array}$ & $\begin{array}{c}-2.99 \\
( \pm 2.59) \\
\end{array}$ & $\begin{array}{c}-1.47 \\
( \pm 0.77) \\
\end{array}$ & $\begin{array}{c}-1.28 \\
( \pm 2.81)\end{array}$ & $\begin{array}{c}1.08 \\
( \pm 2.13)\end{array}$ & $\mathrm{N} / \mathrm{A}$ & $\begin{array}{c}-0.16 \\
( \pm 2.39)\end{array}$ & $\begin{array}{c}-2.35 \\
( \pm 0.94)\end{array}$ \\
\hline
\end{tabular}

Table S11: Temp averages and biases, proportions where AWS-observed southwesterly winds are reported as southeasterly 


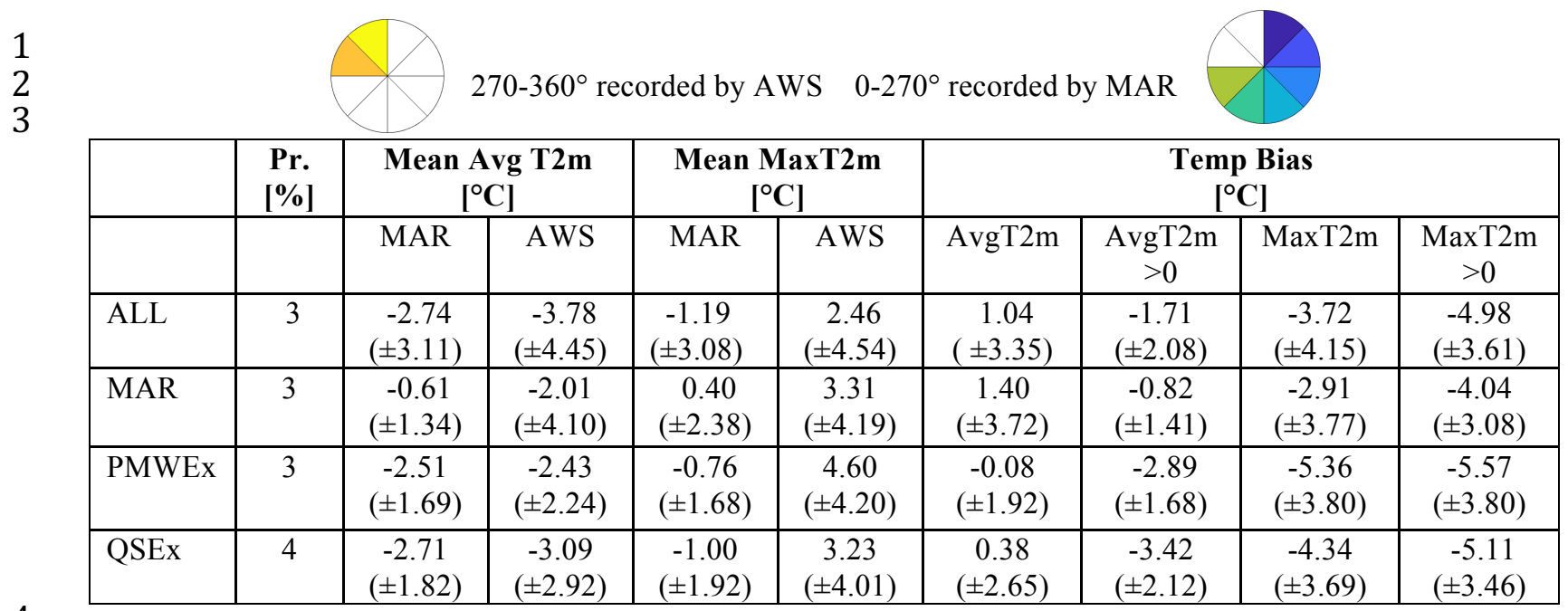

4 Table S12: Temp averages and biases, proportions where AWS-observed southwesterly winds are reported as southeasterly 5 in MAR, as a percentage of all wind direction values for the condition. Conditions for ALL, PMWEx and QSEx (in text) 
1

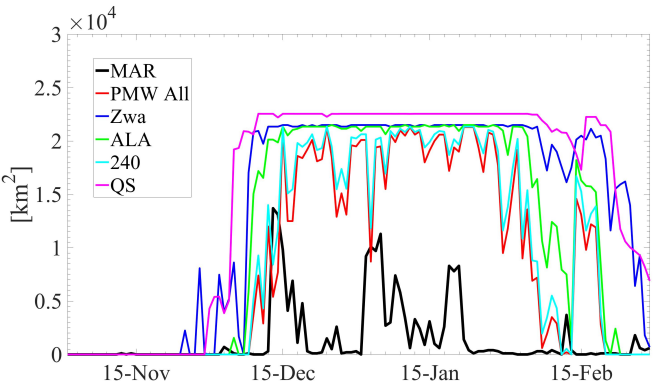

(a) CL melt extent

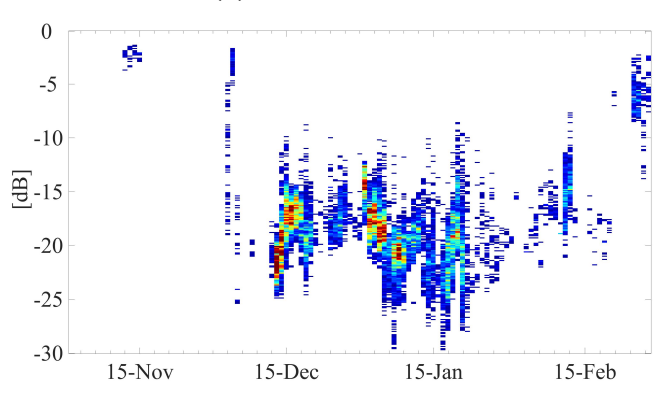

(c)

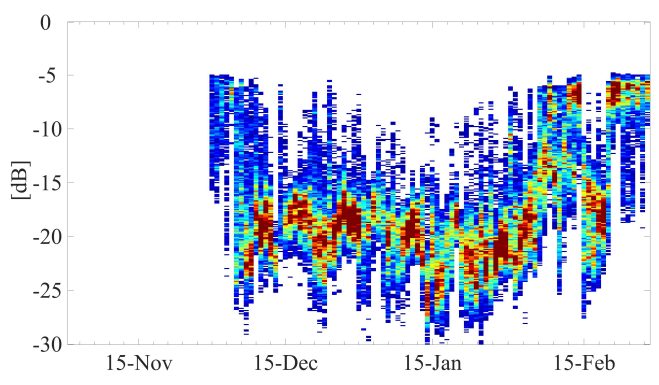

(e)

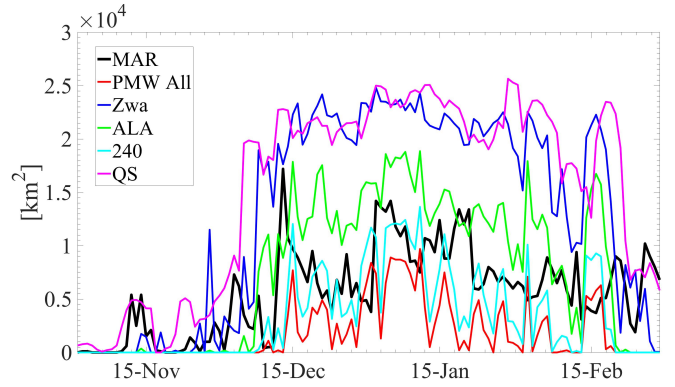

(b) NL melt extent

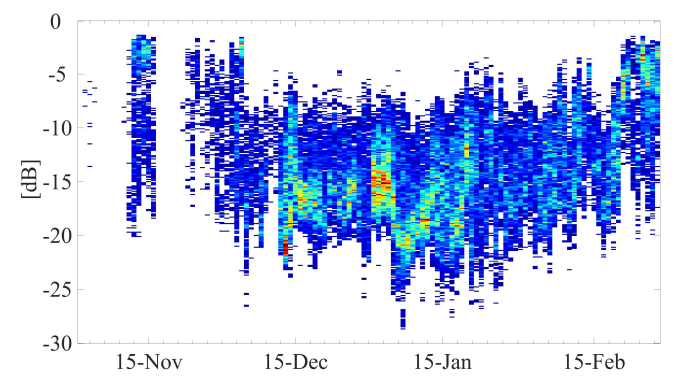

(d)

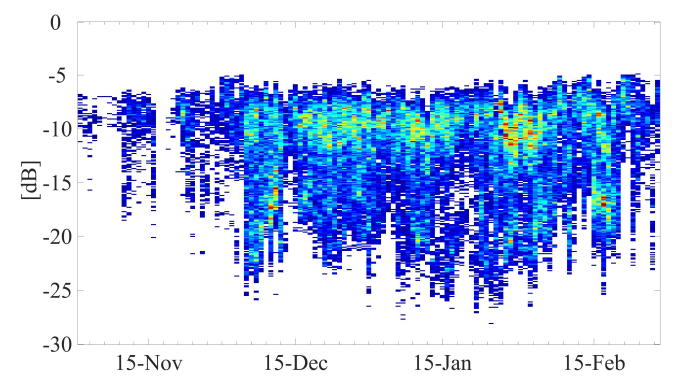

(f)

season., (a) CL region melt extent (b) NL region melt extent. Masks described in text and shown in inset of Fig.2. (c)(d)

14 raw QuikSCAT backscatter for the number of QuikSCAT grid cells $\left(\sim 5 \mathbf{~ k m}^{2}\right)$ where both MAR and QuikSCAT ft3 detect 15 melt (e)(f) raw QuikSCAT backscatter for the number of QuikSCAT grid cells where the QuikSCAT ft3 algorithm 16 detects melt, but MAR does not. 
$\frac{1}{2}$

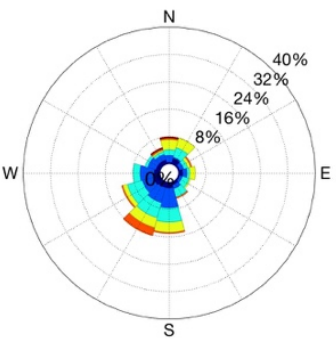

(a) AWS Nov

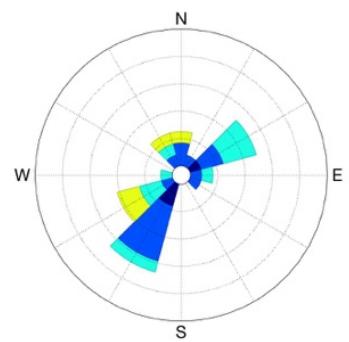

(e) MAR Nov

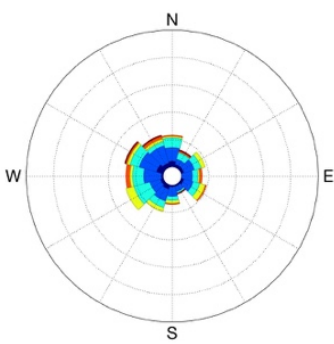

(b) AWS Dec

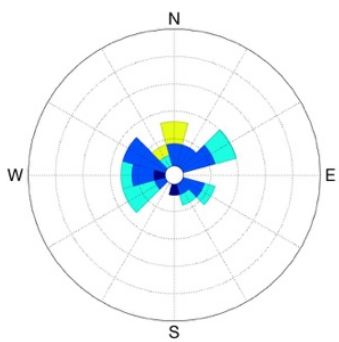

(f) MAR Dec

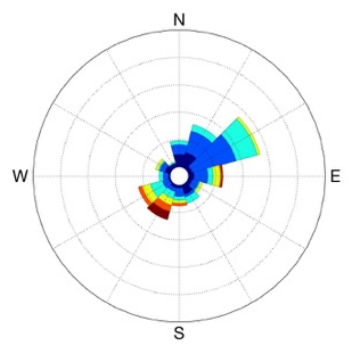

(c) AWS Jan

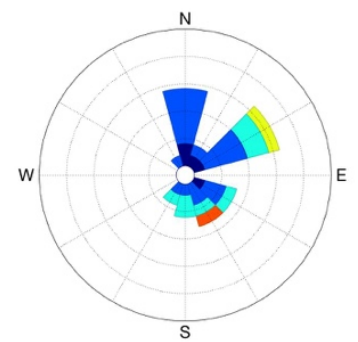

(g) MAR Jan

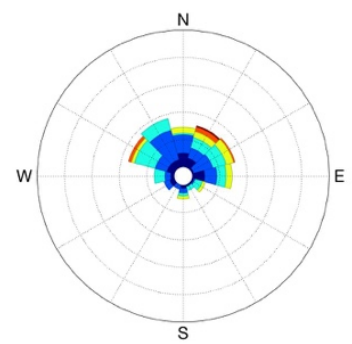

(d) AWS Feb

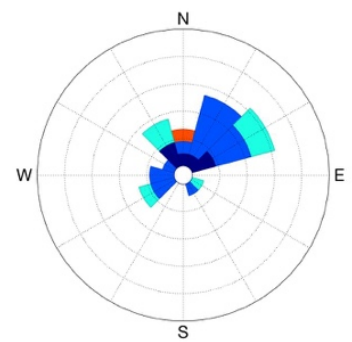

(h) MAR Feb
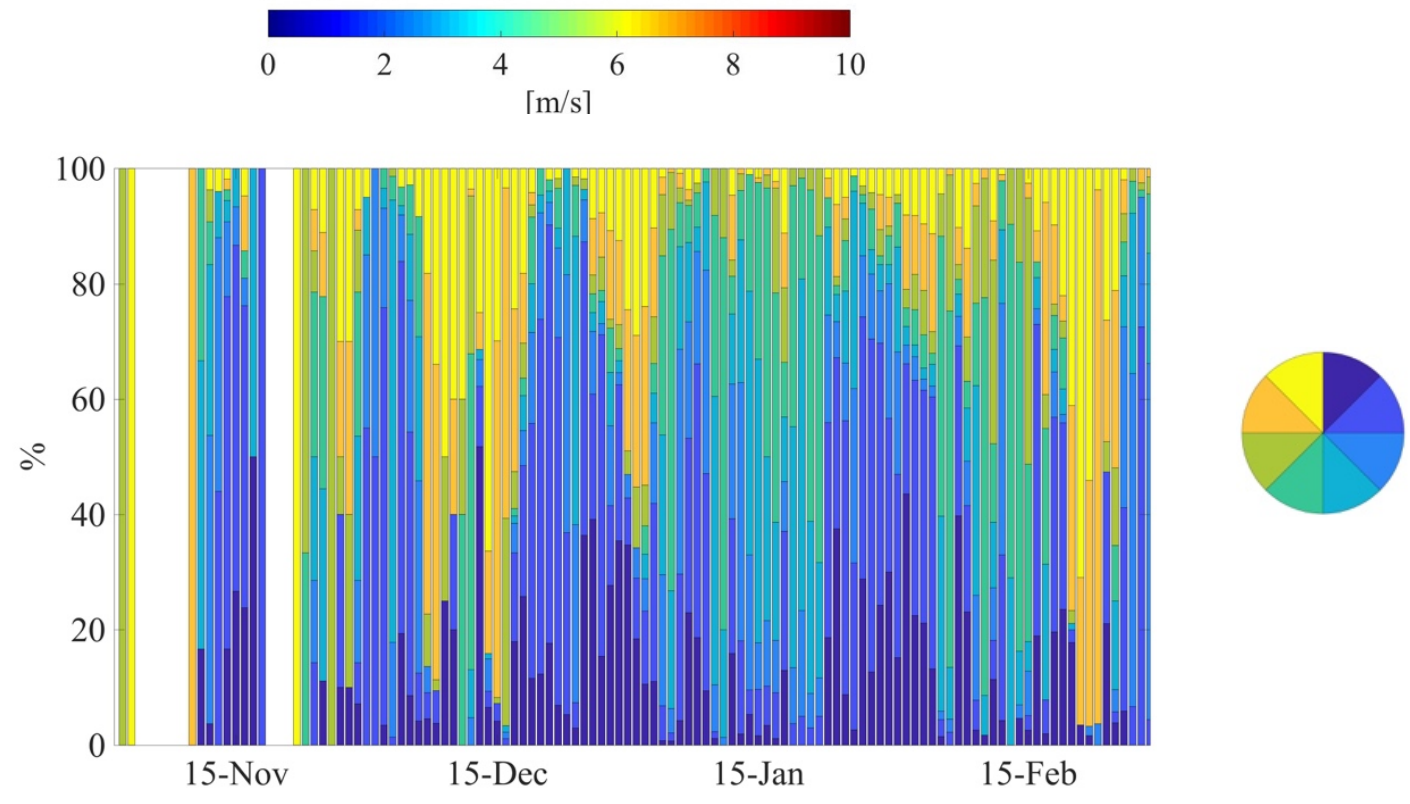

Figure S10 : Wind roses shown for the Larsen IS AWS station in 2001-2002 for (a)Nov (b)Dec (c) Jan (d) Feb. Wind roses shown for the MAR grid cell co-located to the Larsen IS AWS station in 2001-2002 for (e) Nov (f)Dec (g) Jan (h) Feb.(i) Proportion of wind direction (directions shown in inset) for all grid cells where MAR melt occurs in the NL region over the melt season 


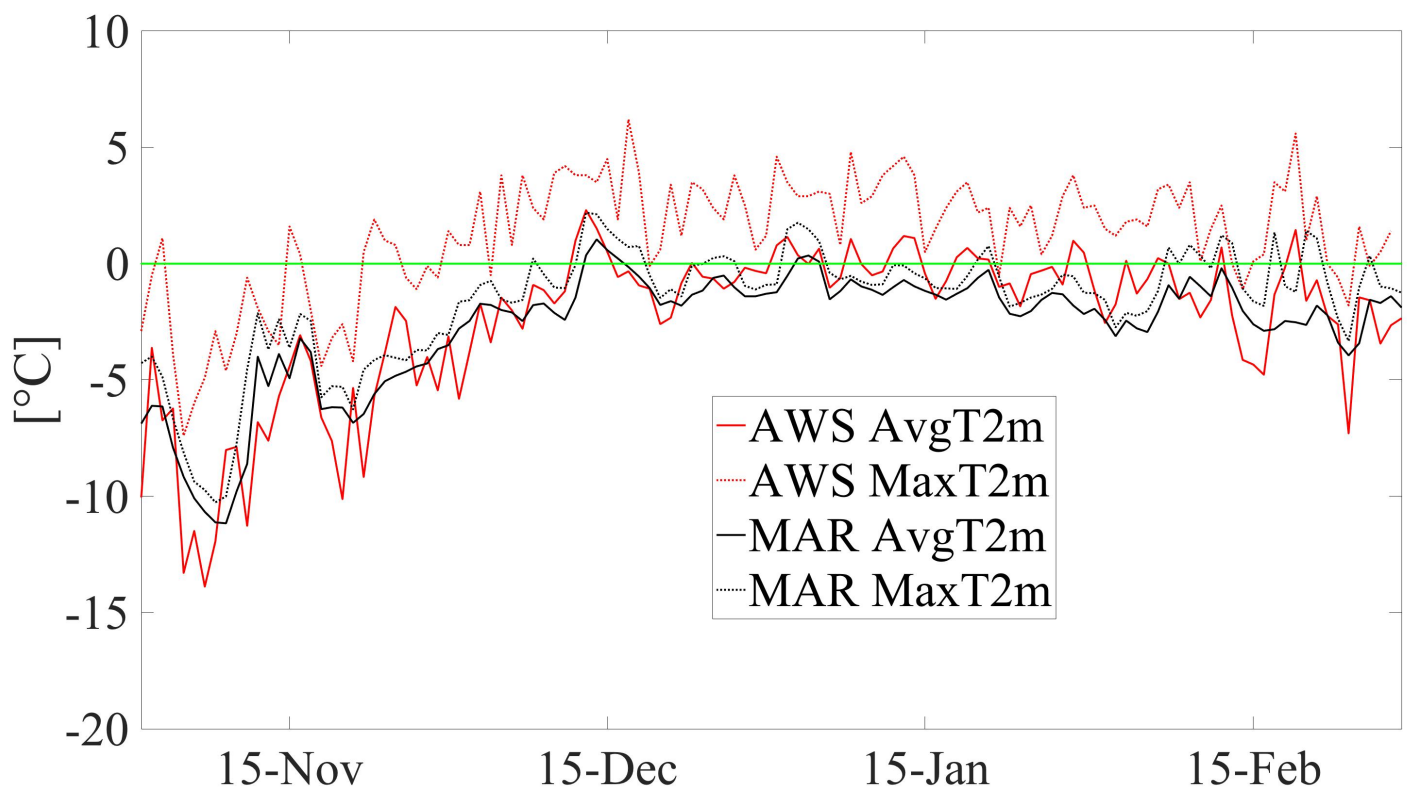

Figure S11: AWS and MAR AvgTs and MaxTs for the 2001-2002 season (Larsen Ice Shelf AWS statio 


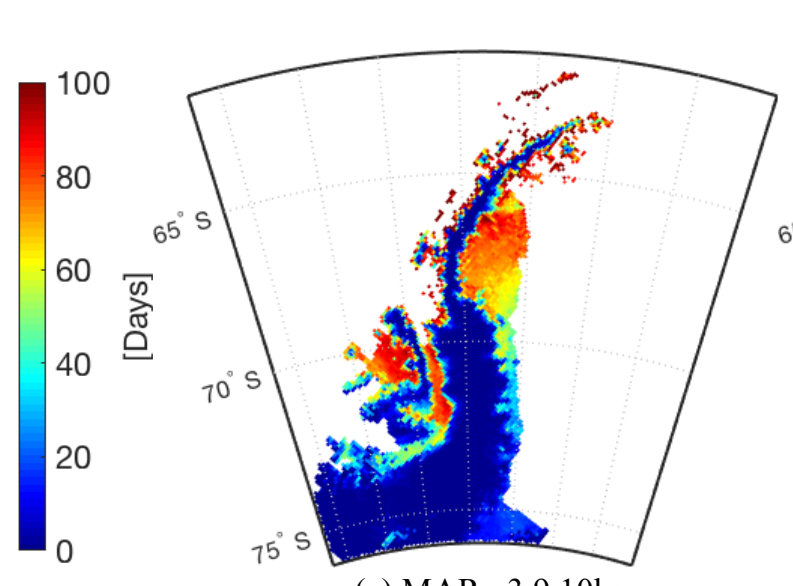

(a) MAR v3.9 $10 \mathrm{~km}$

12

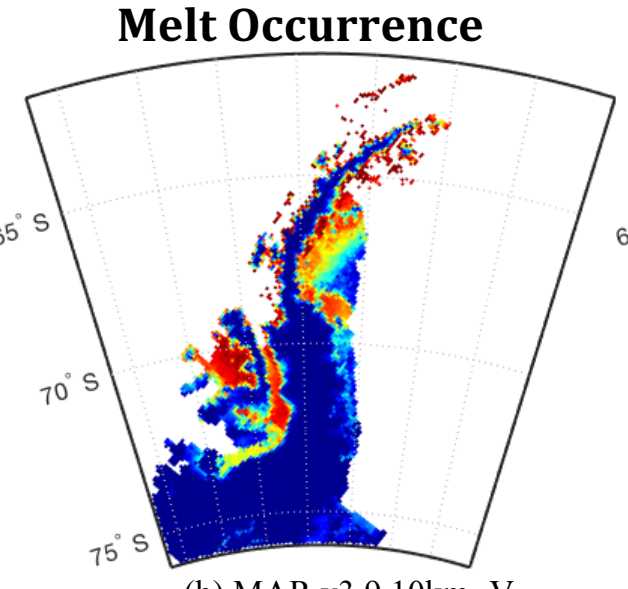

(b) MAR v3.9 $10 \mathrm{~km} \mathrm{~V}$

\section{Meltwater Production}

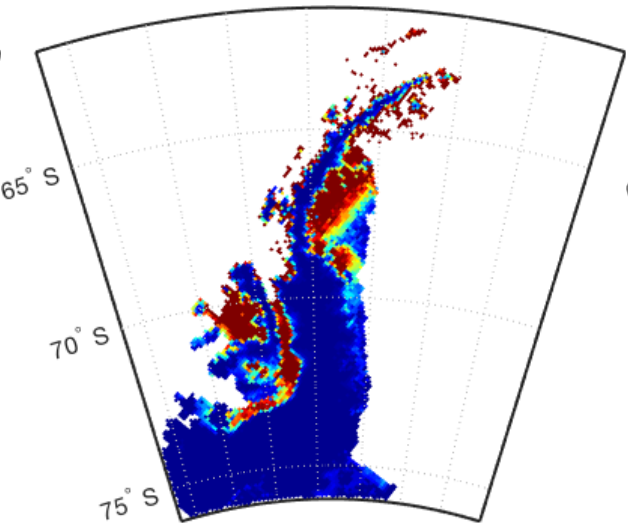

(e) MAR v3.9 10km V

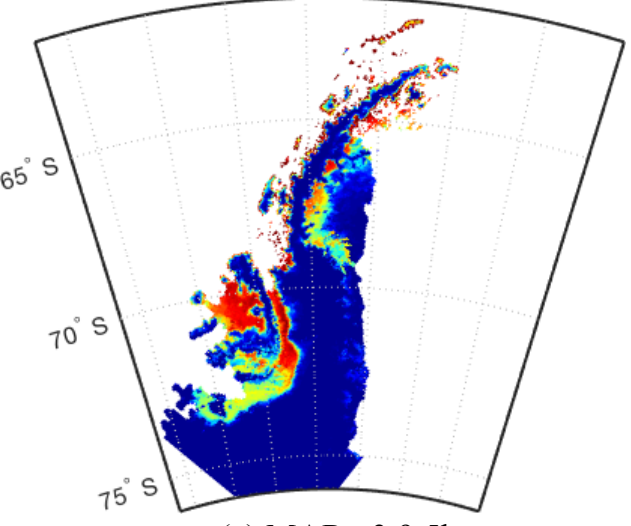

(c) MAR v3.9 5km

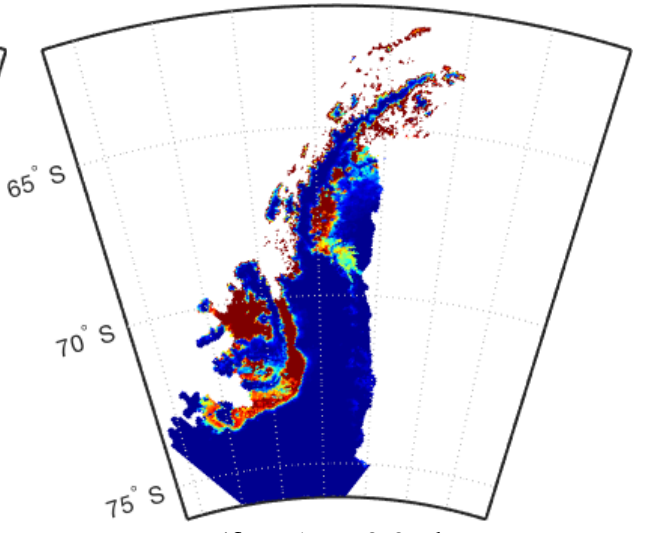

(f) MAR v3.9 5km

Figure S12: Melt occurrence using the $\mathrm{MF}_{0.4}$ criteria (a,b,c) and meltwater production (d,e,f) from Nov 1, 2004 - Feb 28,2005 for MAR v3.9 at (a,d) $10 \mathrm{~km}$ and 23 sigma layers $(b, e) 10 \mathrm{~km}$ and 32 sigma layers and $(c, f) 5 \mathrm{~km}$ horizontal spatial resolution and 23 sigma layers 


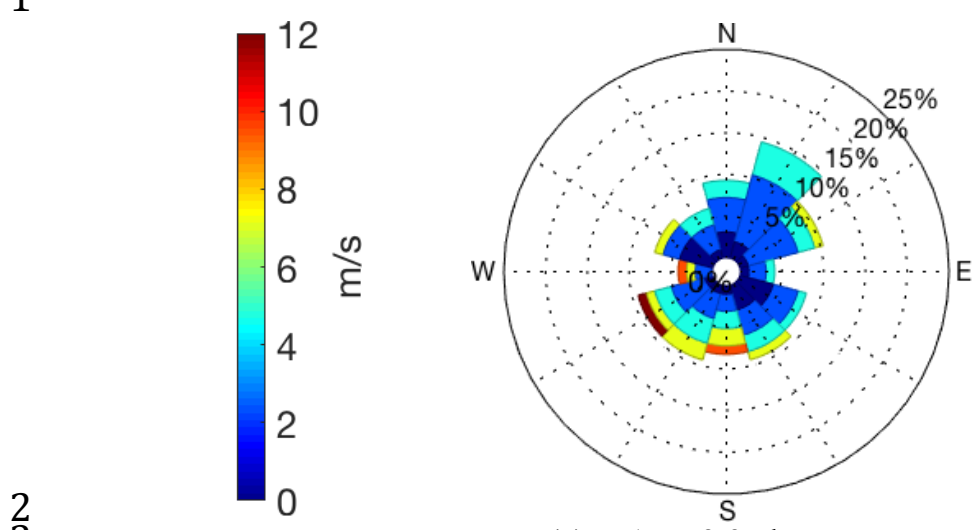

(a) MAR v3.9 5km

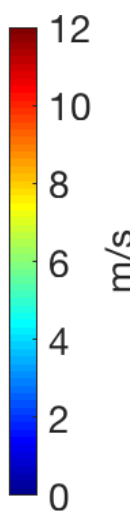

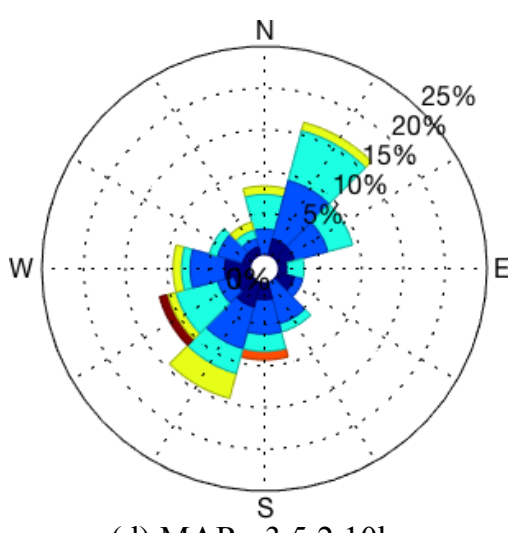

(d) MAR v3.5.2 $10 \mathrm{~km}$ 2005

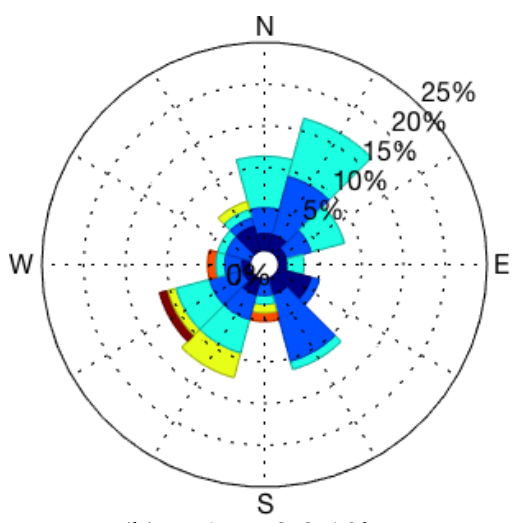

(b) MAR v3.9 10km

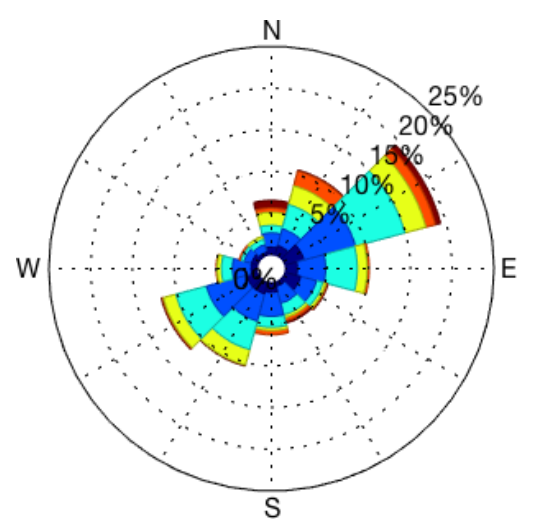

(e) AWS

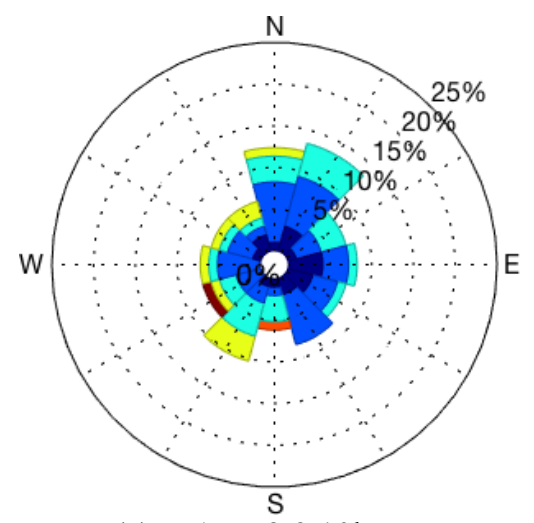

(c) MAR v3.9 10km V

Figure S13: Wind roses at the Larsen IS AWS location (shown in Fig. 1) for Nov 1, 2004 - March 31, 2005 for MAR 3.9 at a $5 \mathrm{~km}$ resolution, 23 sigma layers, daily values (a) $10 \mathrm{~km}$ and 23 sigma layers, daily values (b) $10 \mathrm{~km}$ resolution and 32 sigma layers, daily values (c), MAR 3.5 .2 at a 10km resolution and 23 sigma layers, daily values (d) and AWS at 3-hourly values (e). MAR values are calculated only when AWS data is available and AWS data reports no values between Dec 20, 2004 and February 12, 